\title{
THE VALUE OF KNOWING THE DIRECTION AND NATURE OF THE FORCE CAUSING A FRACTURE
}

\author{
George Perkins, London, England
}

From St Thomas's Hospital

Medical knowledge, expanding from day to day, is becoming so large that we should hesitate to add to the things that have to be learnt, and we may well ask ourselves whether anything is to be gained from knowing the direction and nature of the force causing a fracture. Yet this knowledge is of value, in the first place because we can deduce from it the degree of damage to the soft parts. and in the second place because we can infer from it a logical method of reduction.

The damage sustained by the soft parts often controls treatment. For example, in a crush injury, such as is commonly associated with a motor-cycle accident, the soft tissues suffer much and the skin may be cut or scalped; in a tapping type of injury with little momentum, such as happens when a man is kicked by a horse, the skin is often cut but the soft parts are not otherwise damaged. The difference in the degree of soft-part injury affects immediate treatment. In a crush injury tissues may be devitalised and a wound-toilet operation is often desirable on that account; in a tapping injury there is not any devitalised tissue and a woundtoilet operation is therefore unnecessary. Again, soft parts are not damaged in a spiral fracture and the bone may safely be plated on the day

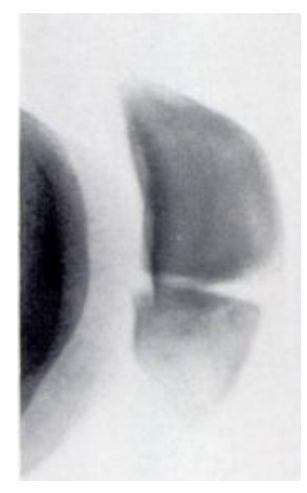

FIG. 1

Fracture of patella, caused by indirect injury. of the accident, whereas in a crush fracture it is advisable to postpone plating for a fortnight.

In the case of a fractured patella the nature of the injuring force decides between two dissimilar treatments. The indirect fracture of the patella occurs when an elderly patient loses his balance. Instinctively he contracts his quadriceps and the extensor apparatus of the knee gives way. The result is a clean transverse fracture of the patella, with rupture of the quadriceps expansion on either side (Fig. 1). From the point of view of treatment the injury ranks as a torn tendon, the ends of which have to be approximated and sewn together. The direct fracture of the patella occurs when a person of any age

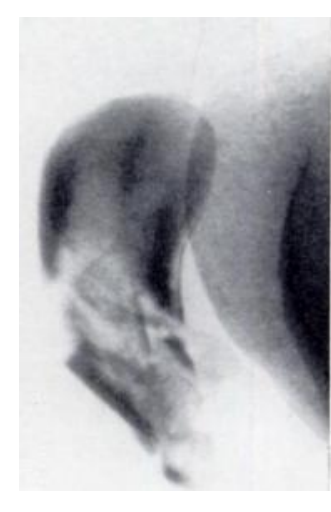

Fig. 2

Fracture of patella, caused by direct injury.

strikes the ground with his knee cap. The patella breaks into several fragments (Fig. 2). There is no muscle damage and the several fragments are held together by the surrounding intact quadriceps, as Furlong says, "like toffee in its paper" (personal communication). Neither muscle nor bone demands treatment and the patient is handled as though he had only sprained his knee. As soon as he can lift his leg off the couch with the knee straight he is encouraged to get up without a splint (Fig. 3).

VOL. 38 B, NO. I, fEBRUARY 1956 


\section{THE EVIDENCE OF THE RADIOGRAPH}

To those who can interpret the signs, the radiographs of a fracture nearly always reveal the direction of the force that broke the bone. A penetrating high-velocity missile has a shattering effect; it behaves as though it exploded after entering the bone, and small fragments are displaced in all directions (Fig. 4). This kind of fracture is rarely seen except during a war. In civilian life the possible forces are longitudinal compression, angulation, shear, transverse compression and rotation.

Longitudinal compression occurs for example when a person falls from a height and lands on his feet. It causes either several longitudinal linear cracks without displacement, or a T-fracture into a joint. The patient shown in Figure 6 fell from a height; he has a fracture of the calcaneum on one side and vertical cracks in the tibia on the other. The patient shown in Figure 5 fell on the point of her flexed elbow.

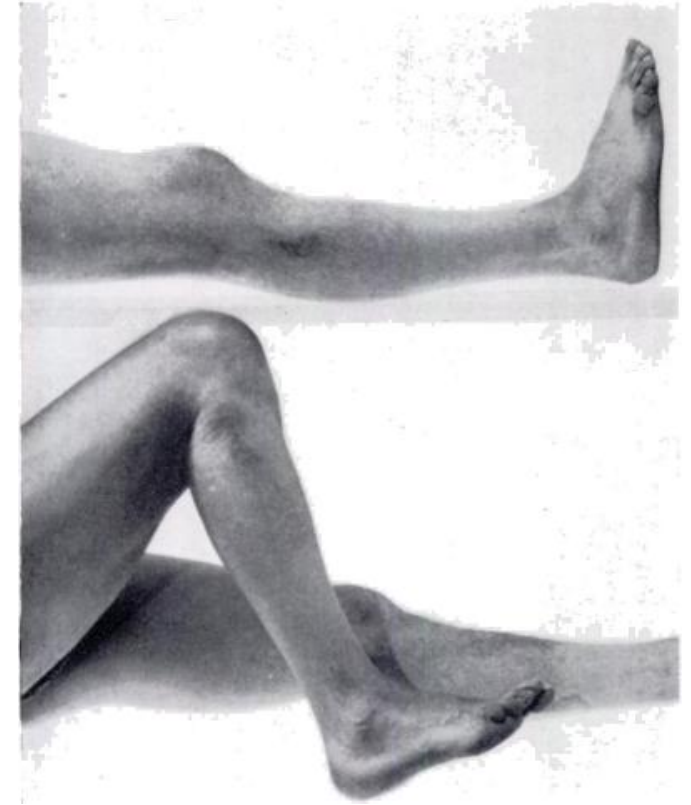

FIG. 3

Range of knee movement four weeks after the fracture shown in Figure 2.

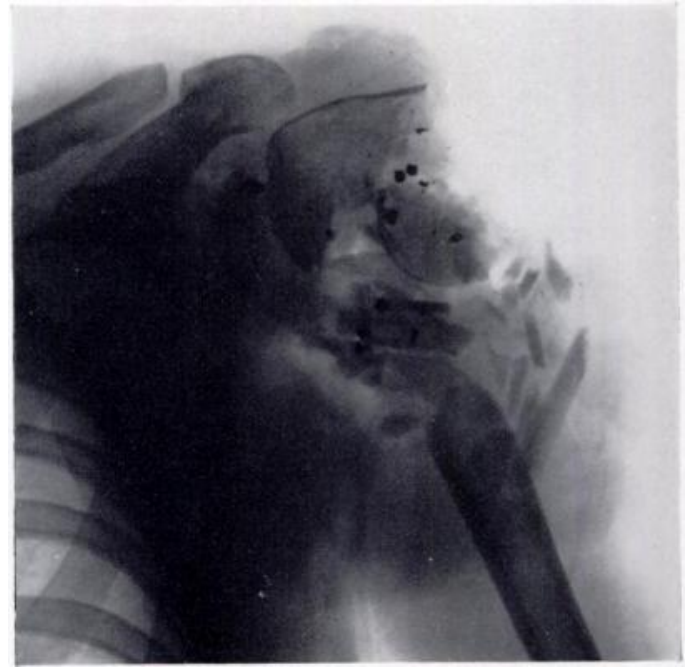

Fig. 4

Gunshot fracture.

The term impaction is much misused. Complete impaction-one end of the bone burying itself in the other-as a result of a compression force is rare, but if the bone is angulated there may be impaction on one side, as in the abduction type of transcervical fracture of the neck of femur (Fig. 7).

An angulation force causes a transverse fracture of the shaft of a bone, often with a separate triangular fragment, the presence of which indicates that the bone was bent until it broke (Fig. 8); but it also shows the direction in which it was bent, for the triangular fragment is always on the inside of the bend. In a child angulation often causes a greenstick fracture.

A force that strikes the shaft of a bone at right angles over a limited area and with dying momentum may be called a tapping force. It causes a clean transverse fracture, often without displacement or at the most with a mild amount of shift. The man shown in Figure 9 was kicked by a horse. With continued momentum in a transverse direction the force may be described as a shearing force (Fig. 19). 

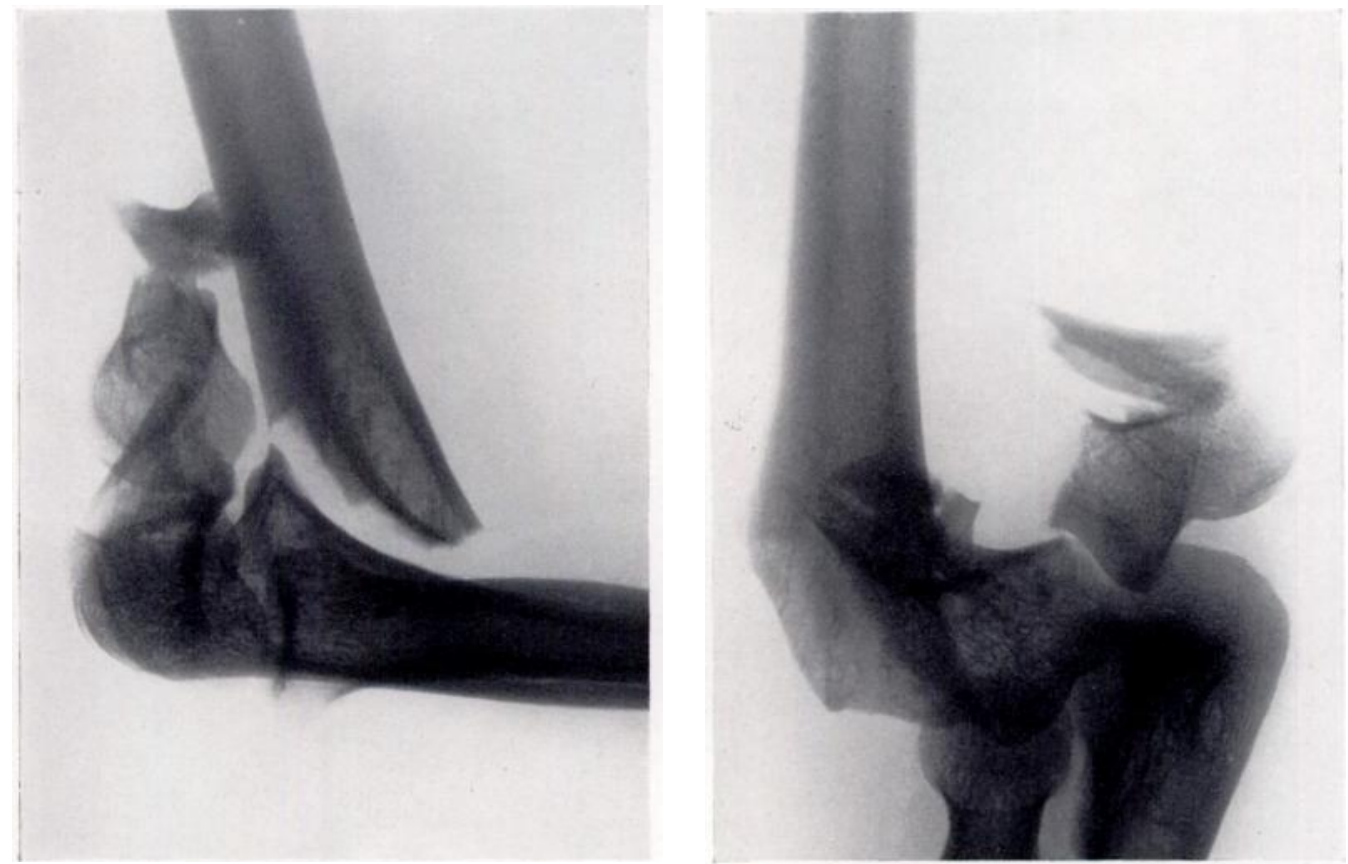

Fig. 5

Longitudinal compression: T-shaped fracture into elbow.

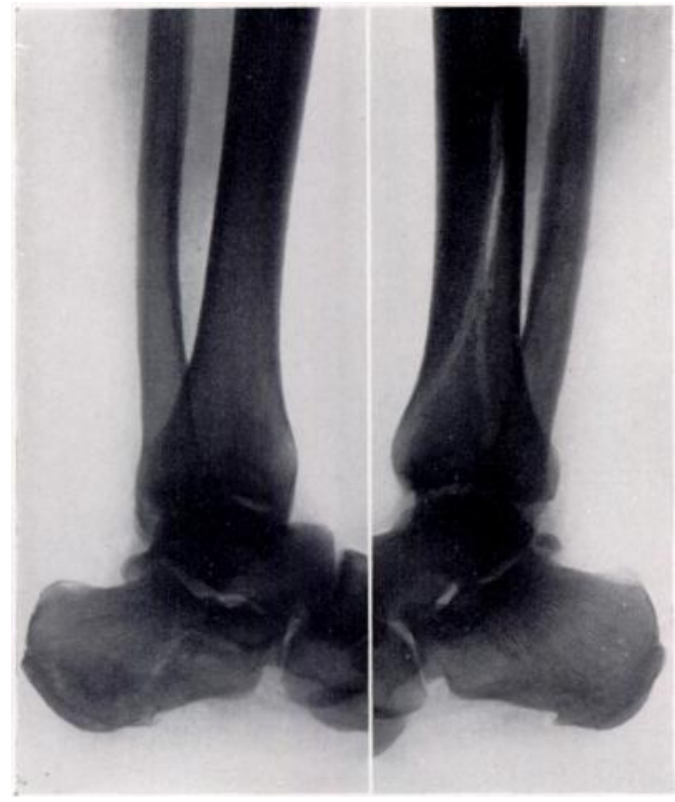

Fig. 6

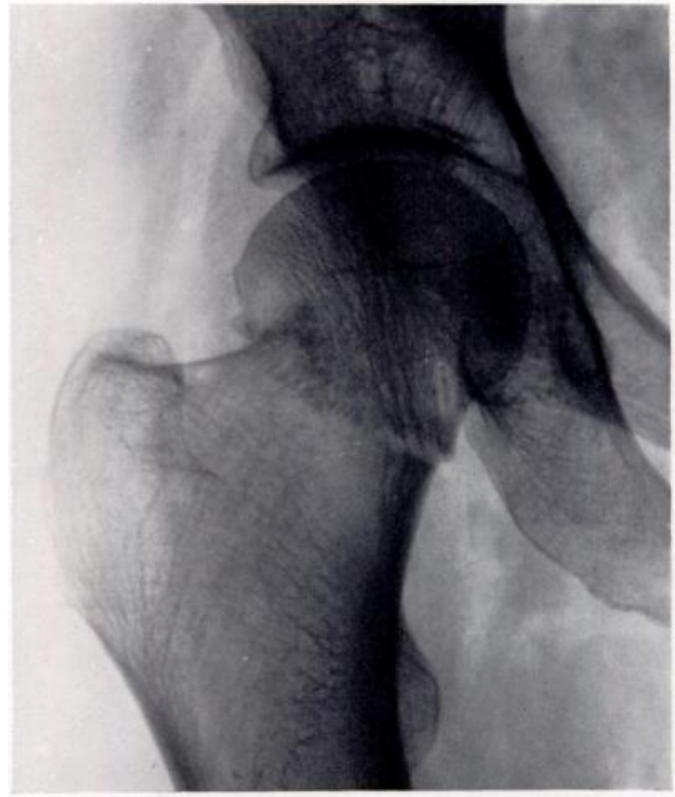

Fig. 7

Figure 6-Longitudinal compression: linear fracture of tibia (R), fracture of calcaneum (L). Figure 7-Angulation force: impacted fracture of the neck of the femur.

VOL. 38 B, NO. 1, FEBRUARY 1956 
Transverse compression occurs when a limb is crushed. The nature of the injuring force is self-evident when a bus wheel runs over a leg or a man is pinned under a collapsed building; but crushing is also present in many motor car accidents. The crushing element is important, for the soft tissues are damaged as well as the bones, and the degree of damage to the soft parts often controls the immediate treatment. It was possible to deduce from Figure 10 that the tissues had been crushed; plating was therefore postponed for fourteen days, the patient meanwhile being made comfortable by skeletal traction. Several days after the accident the damage to the soft parts became self-evident (Fig. 11).

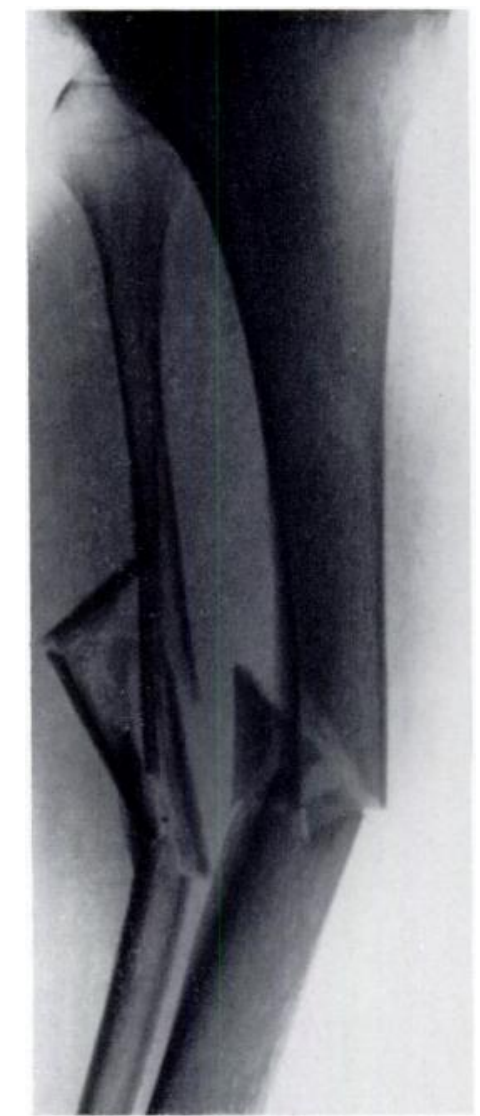

FIG. 8

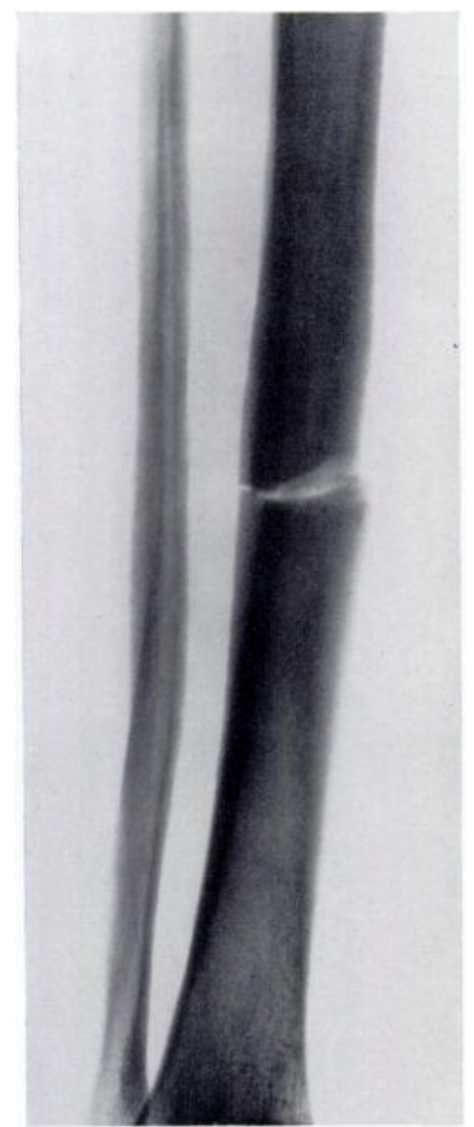

FIG. 9

Figure 8-Angulation force: transverse fractures of the tibia and fibula, at the same level. Figure 9-Tapping force: transverse linear fracture of the tibia.

Rotation causes a spiral fracture. The fracture is restricted to the diaphysis but extends over a considerable stretch of the shaft. The common fracture of the shaft of the femur in children is produced by rotation. In the adult, rotation damages the leg below the knee more often than the thigh; the tibia is broken in the lower third and the fibula in the upper third, and both fractures have the characteristics of a spiral fracture (Fig. 12). An oblique fracture is a variant of the transverse fracture, and is caused either by angulation or by shear. In the oblique fracture it is possible by taking several radiographs to find one that shows a gap throughout the whole extent of the fracture. In a spiral fracture it is not possible to get a radiograph that shows a complete gap unless the fragments are widely separated. Figure 13 at first sight looks like a spiral fracture; it is in fact an oblique transverse fracture. 


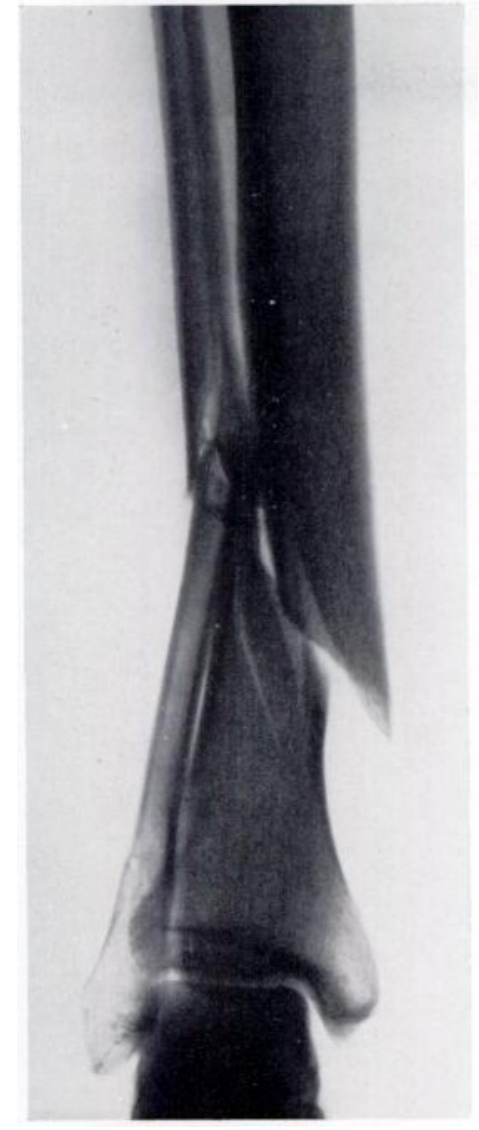

Fig. 10

Crushing force: oblique fractures of tibia and fibula at the same level.

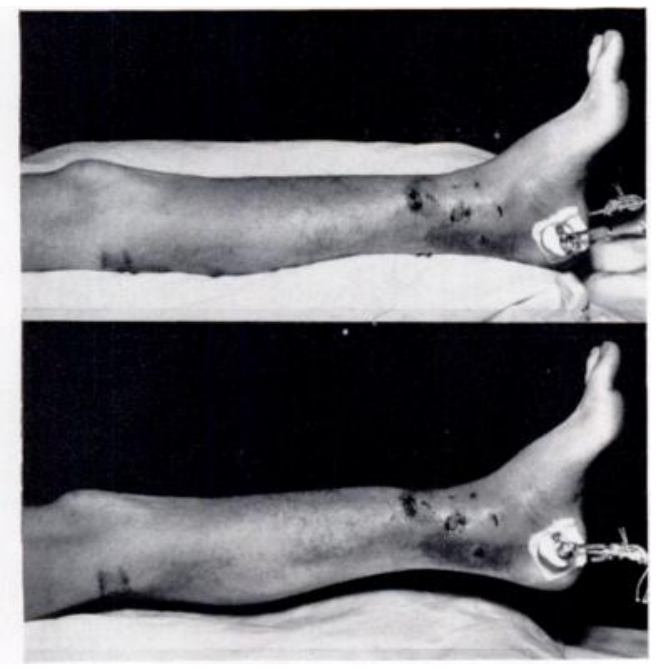

Fig. 11

Seven days after the fracture shown in Figure 10. Patient lifting his leg off the bed. Note injury to the soft parts.

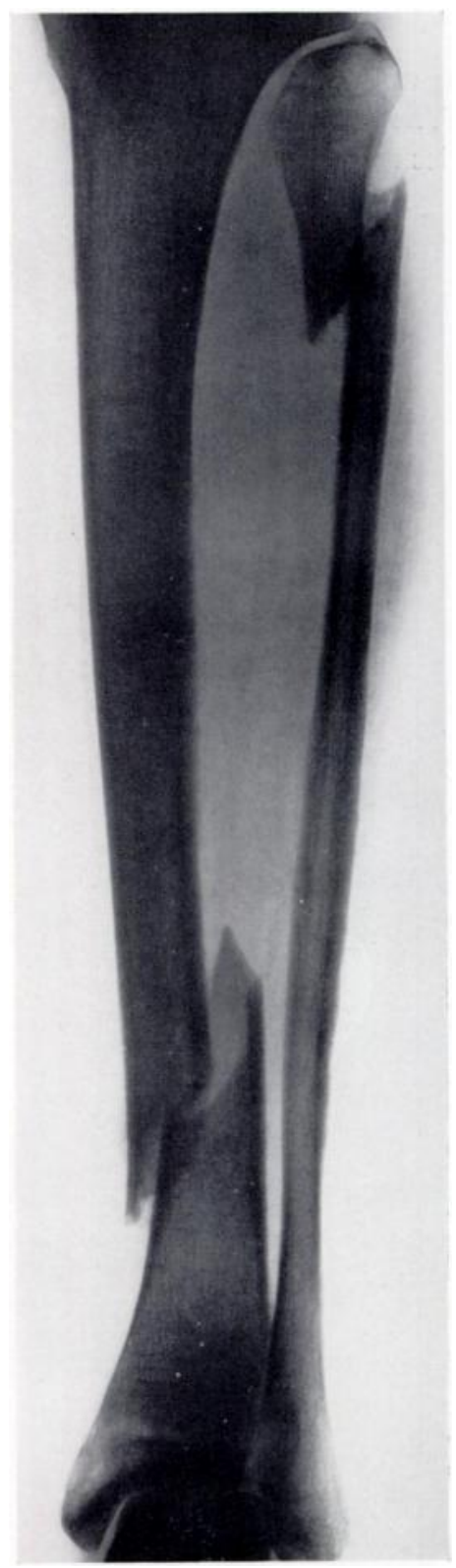

FIG. 12

Twisting force: spiral fractures of the tibia and fibula at different levels.

VOL. 38 B, NO. 1, FEBRUARY 1956 


\section{OTHER EVIDENCE}

In addition to radiography there are other pointers to the nature of the fracturing force.

The history is important but it may be equivocal. A person falling from a height and landing on his feet has obviously been exposed to a longitudinal compression. When, however, a child falls on to its outstretched hand and sustains fractures of the shafts of the radius and ulna, a rotational force in addition is involved, for as the child strikes the ground the trunk twists on the fixed hand.

The nature of the skin damage is informative. After a head injury a graze on the face implies that the patient came to rest by sliding rather than by butting something head on, and damage to the brain is not likely to be serious. A wound on the forehead makes one suspect a hyperextension injury to the neck. And experience tells us that if a patient has hurt his foot treading off the pavement he has in all probability sustained a lateral-rotation Pott's fracture.

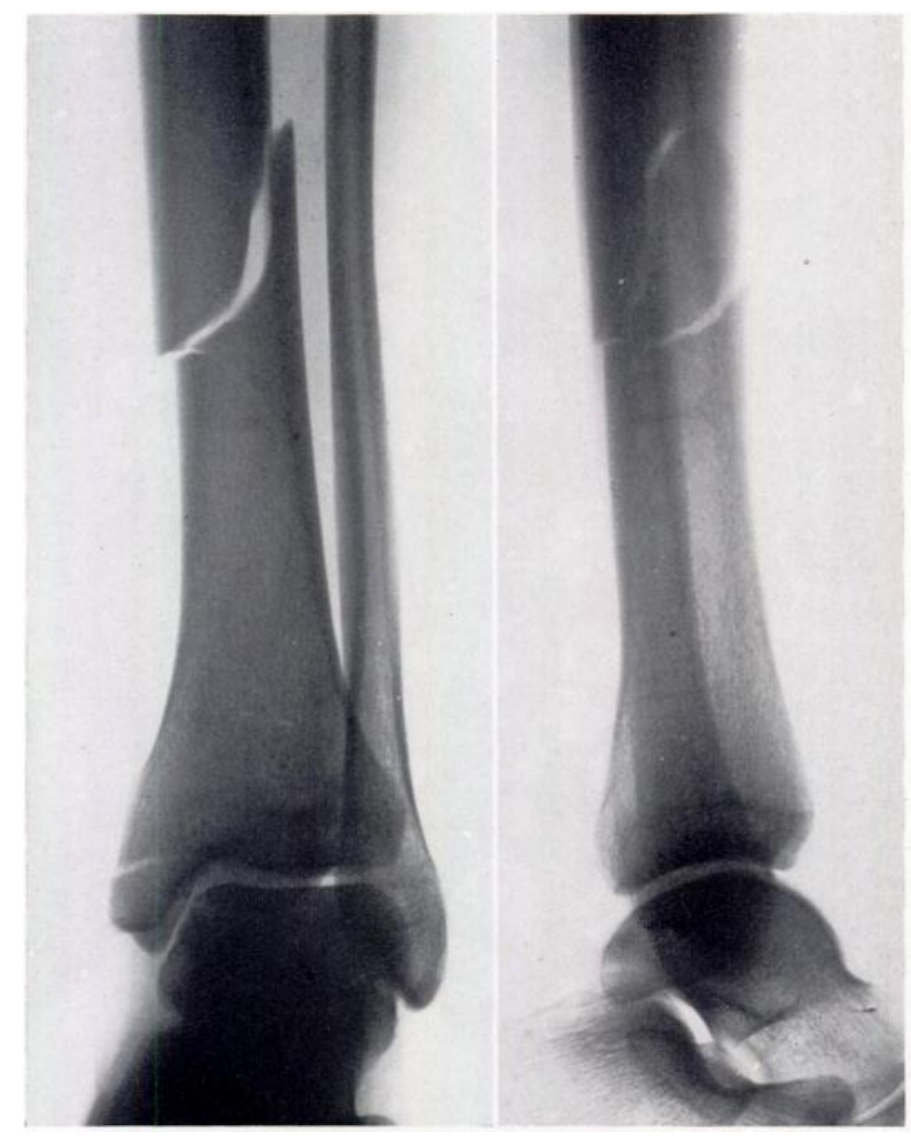

Fig. 13

Oblique fracture mimicking a spiral fracture.

In many fractures a knowledge of the direction of the fracturing force points out an obvious and easy method of reduction. For example, in a Colles's fracture the lower fragment has been tilted backwards by an angulation force and, at the same time, it and the hand have been supinated on the ulna. To correct the displacement an opposite force is applied: the doctor's two thumbs are placed on the back over the lower fragment of the radius, and the lower fragment is tilted forwards and pronated (Fig. 14). As another example Mervyn Evans (1951) has pointed out that fractures of the forearm caused by a fall on the hand can be reduced by supinating the forearm. 


\section{THE FORCES CAUSING POTT'S FRACTURES}

A Pott's fracture best illustrates the usefulness of knowing how a bone has been broken. The fracture Pott described is different from the fracture he sustained, and the exact nature of both is uncertain; but it is convenient, clinically, to use the term Pott's fracture to cover any fracture of the leg bones involving the ankle joint.

It is customary to describe degrees of Pott's fracture. This is to be deprecated on two accounts. First, there is not any point in distinguishing a fracture as first, second or third degree unless the method of treatment is different; and second, the terminology gives one no clue to the direction of the fracturing force and therefore no clue to the force required to reduce the displacement.

Subdividing Pott's fracture according to the direction of the fracturing force we have the following varieties: lateral-rotation, abduction, adduction, shear and vertical. And in nearly every case radiography enables the doctor to guess correctly which fracture he has to deal with.

Lateral-rotation causes a fracture through the lateral malleolus. The fracture is often invisible in the antero-posterior radiograph; in the lateral it appears as a line, more vertical

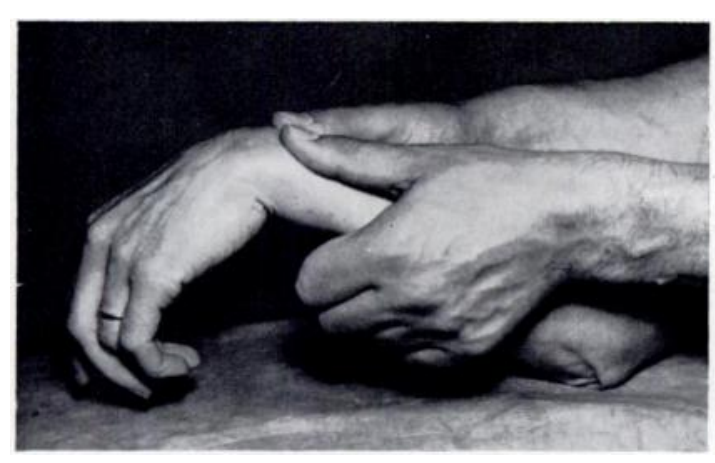

FIG. 14

Method of reducing Colles's fracture.

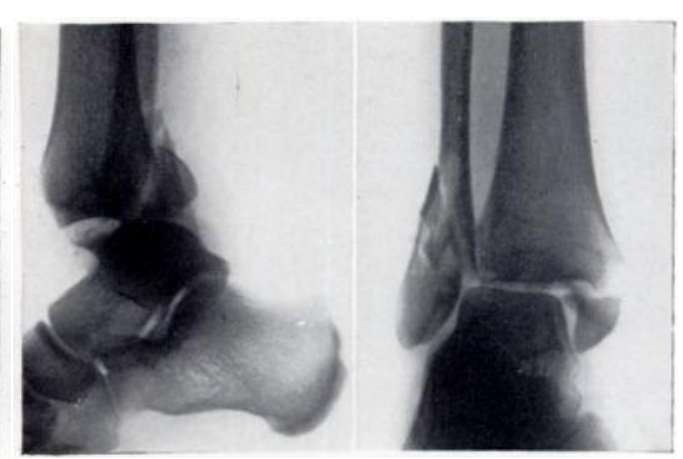

Fig. 15 Lateral-rotation Pott's fracture.

than horizontal, which begins below at the level of the mortise and runs upwards and backwards (Fig. 15). The medial malleolus may or may not be broken; if it is, the antero-posterior radiograph shows a transverse fracture below the level of the horizontal part of the ankle mortise. The situation of this fracture is important because the portion of the medial malleolus left attached to the shaft acts as a buffer against which the talus can be reduced. The lateral malleolar fragment is displaced backwards; when the displacement is great the posterior third of the articular surface also splits off from the tibia. This fracture is seen as a vertical line in the lateral view. In most lateral-rotation Pott's fractures the displacement is slight; only when the displacement is great does it reveal clearly that rotation has been the essential fracturing force (Fig. 16).

A lateral-rotation fracture is reduced by rotating the foot inwards. Exact replacement of the bones is not necessary provided that the talus has been restored to its original bed in the ankle mortise. As in all Pott's fractures, subluxation of the ankle, not the displacement of the bones, is the important fact and treatment is centred on restoring the talus to its proper position. The amount of displacement of the talus varies from a slight lateral shift or a slight lateral tilt to almost a dislocation. But whatever the amount of the displacement, the method of reduction is the same. There is no point therefore in subdividing a Pott's fracture into degrees, as is often done.

VOL. 38 B, NO. 1, FEBRUARY 1956 
The abduction fracture is not as common as the lateral-rotation fracture, but is more important because it is much more likely to leave the ankle crippled. In the antero-posterior radiograph there is a transverse fracture of the fibula about two inches up (Fig. 17). Often a small intermediate fragment indicates by its triangular shape that the break was caused by angulation. The medial malleolus may escape or it may break at the same level as in the lateral-rotation fracture.

Displacement in an abduction fracture is corrected by adducting the foot. To make the reduction complete considerable force is required. One need not be afraid of over-reduction because the small fragment of the medial malleolus that remains attached to the shaft acts as a buttress to prevent the talus from overshooting the mortise.

The abduction fracture, being horizontal, is prone to angulate when the patient starts to bear weight. For this reason an abduction Pott's fracture needs protection while it is proceeding from union to consolidation, whereas in most other Pott's fractures splintage can safely be discarded as soon as the bones have joined.
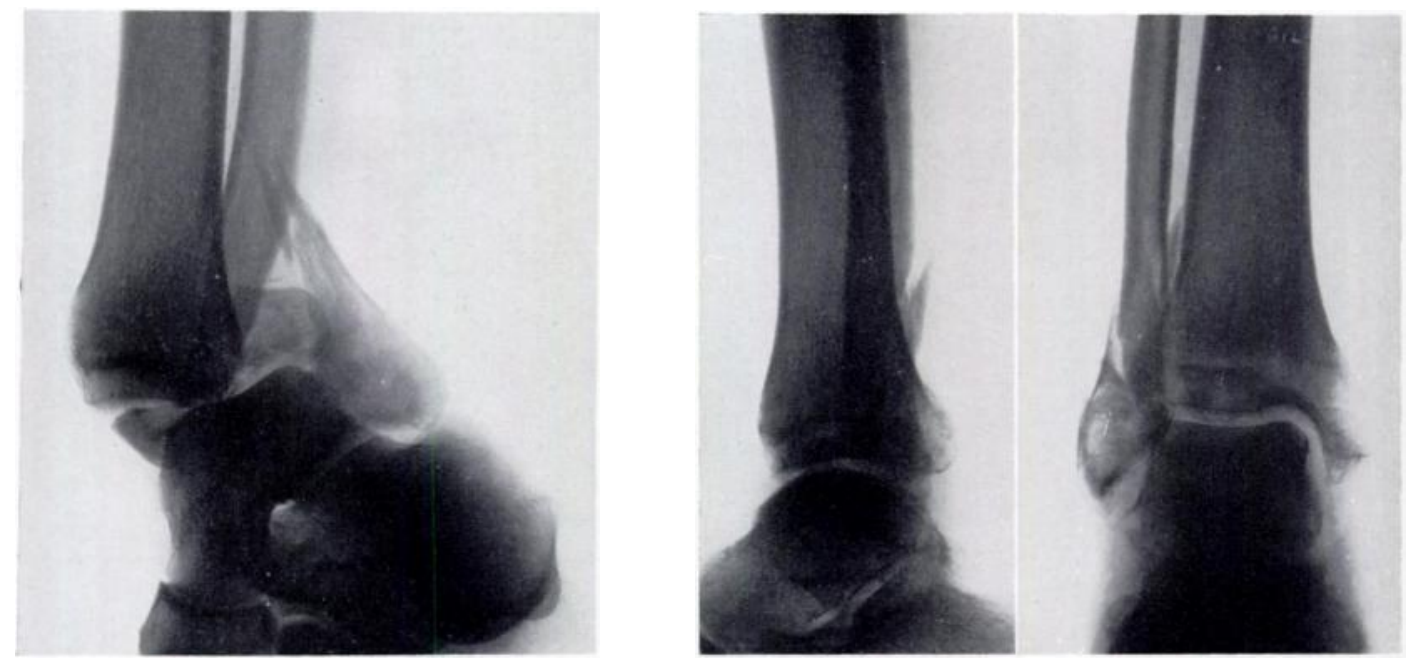

Fig. 16

Lateral-rotation Pott's fracture before and after reduction.

An adduction force on the foot causes as a rule a sprain of the subtalar joint (the common misnamed sprain of the ankle). Should the force continue, the tibia breaks at its junction with the medial malleolus. The radiograph is distinctive (Fig. 18). The fracture is in the sagittal plane. Starting below at the junction of the horizontal and vertical parts of the mortise it runs almost vertically upwards, emerging on the medial border of the tibia. The tip of the lateral malleolus is often avulsed but the malleolus itself does not break as a rule.

An adduction fracture is reduced by abducting the foot. (The terminology is confusing, for abduction and adduction of the foot are usually referred to as inversion and eversion.)

The shear fracture is not common. It is caused by a force that would elsewhere result in a crush fracture. Both malleoli break transversely at the level of the horizontal part of the mortise. The foot is displaced medially or laterally according to the direction of the force (Fig. 19). The site of the malleolar fractures makes any reduction unstable on account of the absence of side buttresses to hold the talus. In practice stability is obtained by screwing the medial malleolus to the shaft of the tibia. The medial malleolus then acts as a buffer against which the talus is pushed. The shear fracture is mimicked on occasions by a lateral-rotation fracture (Fig. 20). The essence of the shear is that both malleoli break at their junction with the shafts, and this renders closed reduction unstable. 


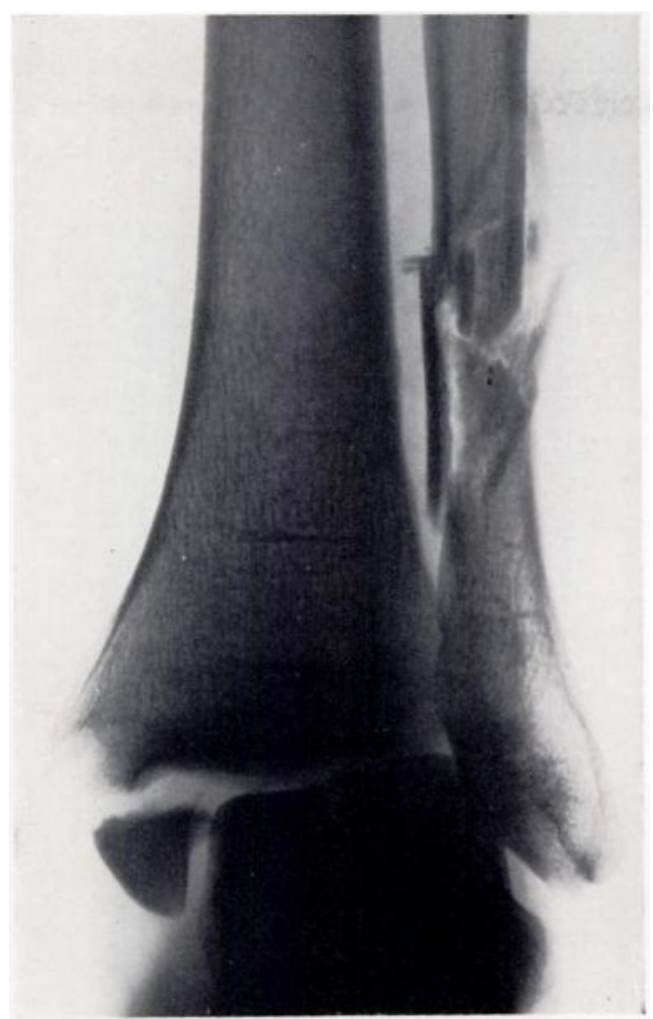

Fig. 17

Abduction Pott's fracture.

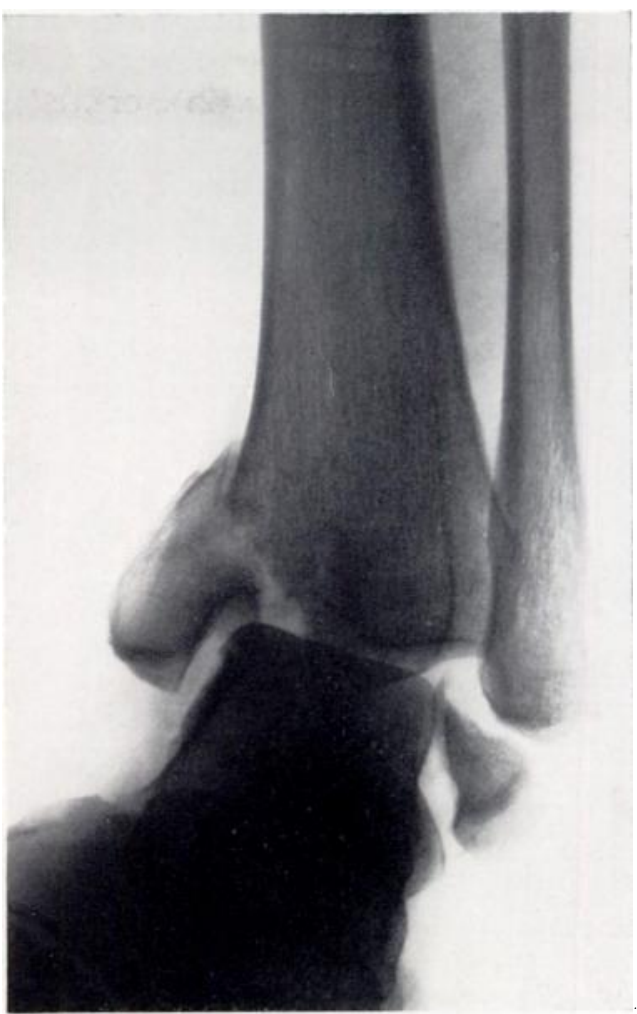

FIG. 18

Adduction Pott's fracture.

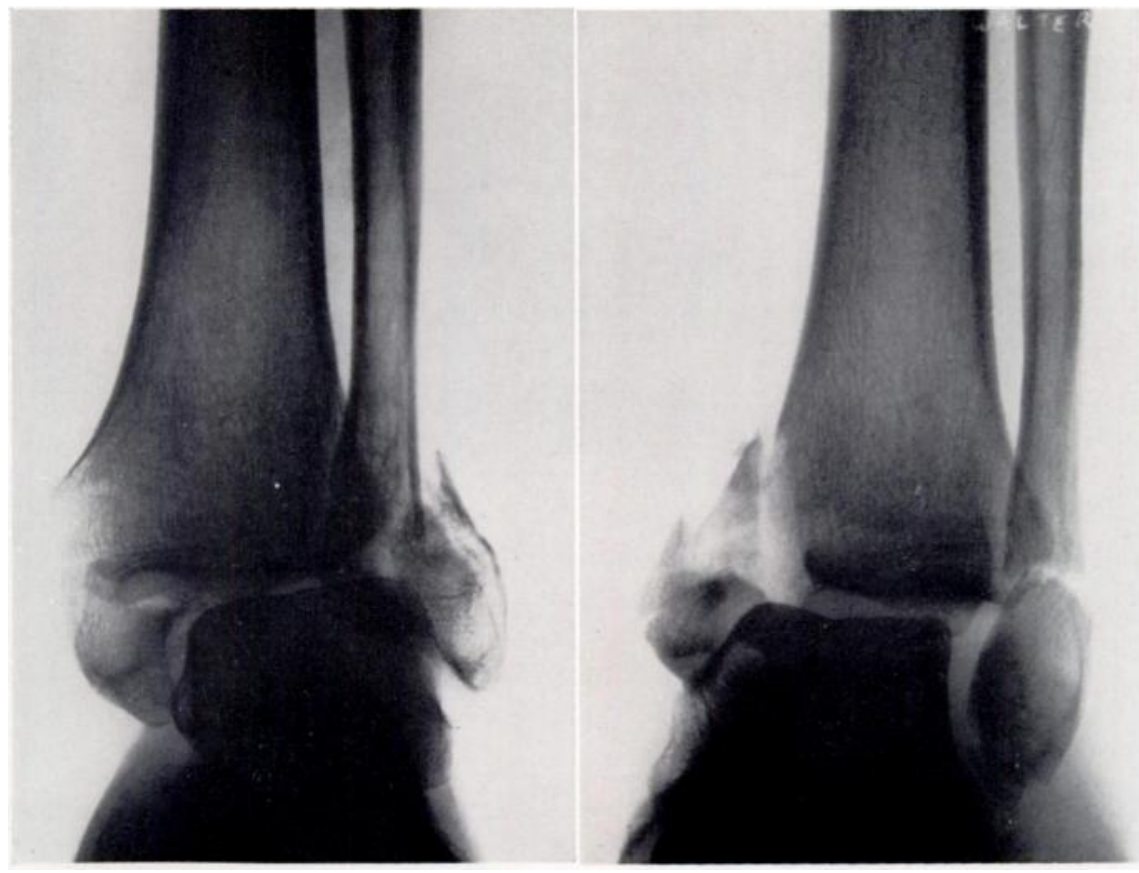

FIG. 19

Shearing Pott's fractures. Left: Talus shifted laterally. Right: Talus shifted medially. VOL. 38 B, NO. 1, FEBRUARY 1956 
The vertical fracture is caused by longitudinal compression, and the usual history is a fall from a height. It causes either $a$ ) a T-shaped fracture of the lower end of the tibia with marked displacement (Fig. 21), or b) linear cracks running up the shaft without any displacement (Fig. 6). The latter is a trivial injury; it requires little or no treatment and recovery is speedy. The former is a serious injury and leaves a permanent disability. In view of the direction of the fracturing force skeletal traction is applied through the calcaneum. This

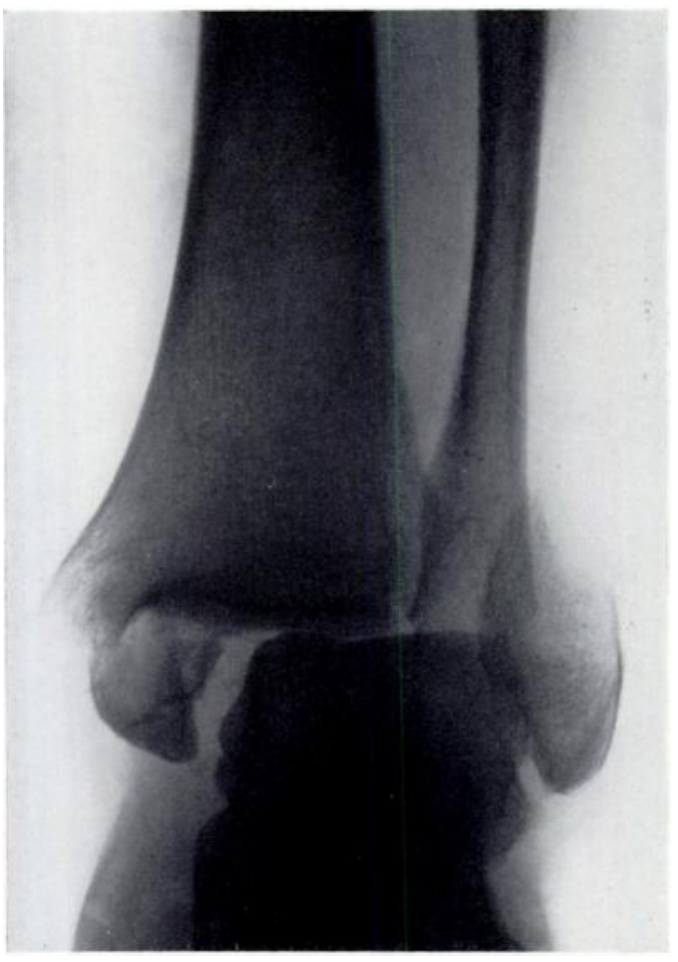

FIG. 20

Lateral-rotation Pott's fracture, mimicking a shearing fracture.

is a good method of treatment because it enables the patient to start moving his ankle at once. Skeletal traction does not always produce anatomical reduction and the lateral radiograph may show a step in the articular surface of the tibia, but by moving the ankle early the patient renders congruous the opposing articular surfaces of the tibia and talus (Fig. 22). It should be noted that skeletal traction is only of use in the treatment of the vertical type of Pott's fracture. It does not, for example, reduce a lateral-rotation fracture. In order to obtain reduction of a fracture, force should be applied in a direction opposite to that causing the fracture. This is the main reason why it is helpful to work out the nature and direction of the blow. And these can be deduced from the story of the accident, the damage to the skin, and the radiographs.

\section{REFERENCE}

Evans, E. M. (1951): Fractures of the Radius and Ulna. Journal of Bone and Joint Surgery, 33-B, 548. 